\title{
Pharmacogenomic analysis of patient- derived tumor cells in gynecologic cancers
}

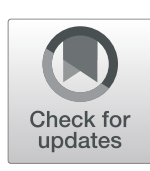

Jason K. Sa ${ }^{1,2,3 \dagger}$, Jae Ryoung Hwang ${ }^{4 \dagger}$, Young-Jae Cho ${ }^{5 \dagger}$, Ji-Yoon Ryu ${ }^{5}$, Jung-Joo Choi ${ }^{5}$, Soo Young Jeong ${ }^{5}$, Jihye Kim ${ }^{6}$, Myeong Seon Kim5, E. Sun Paik ${ }^{5}$, Yoo-Young Lee ${ }^{5}$, Chel Hun Choi ${ }^{5}$, Tae-Joong Kim ${ }^{5}$ Byoung-Gie Kim², Duk-Soo Bae ${ }^{5}$, Yeri Lee ${ }^{1,2}$, Nam-Gu Her ${ }^{1,2}$, Yong Jae Shin ${ }^{1,2,7}$, Hee Jin Cho ${ }^{1,2}$, Ja Yeon Kim ${ }^{1,2}$, Yun Jee Seo ${ }^{1,2}$, Harim Koo ${ }^{1,8}$, Jeong-Woo Oh ${ }^{1,8}$, Taebum Lee ${ }^{9}$, Hyun-Soo Kim ${ }^{10}$, Sang Yong Song ${ }^{10}$, Joon Seol Bae ${ }^{11}$, Woong-Yang Park ${ }^{11}$, Hee Dong Han ${ }^{12}$, Hyung Jun Ahn ${ }^{13}$, Anil K. Sood ${ }^{14}$, Raul Rabadan ${ }^{15,16}$, Jin-Ku Lee ${ }^{17^{*}}$ (D, Do-Hyun Nam ${ }^{1,7,8^{*}}$ and Jeong-Won Lee ${ }^{1,5,8^{*}}$

\begin{abstract}
Background: Gynecologic malignancy is one of the leading causes of mortality in female adults worldwide. Comprehensive genomic analysis has revealed a list of molecular aberrations that are essential to tumorigenesis, progression, and metastasis of gynecologic tumors. However, targeting such alterations has frequently led to treatment failures due to underlying genomic complexity and simultaneous activation of various tumor cell survival pathway molecules. A compilation of molecular characterization of tumors with pharmacological drug response is the next step toward clinical application of patient-tailored treatment regimens.
\end{abstract}

Results: Toward this goal, we establish a library of 139 gynecologic tumors including epithelial ovarian cancers (EOCs), cervical, endometrial tumors, and uterine sarcomas that are genomically and/or pharmacologically annotated and explore dynamic pharmacogenomic associations against 37 molecularly targeted drugs. We discover lineage-specific drug sensitivities based on subcategorization of gynecologic tumors and identify TP53 mutation as a molecular determinant that elicits therapeutic response to poly (ADP-Ribose) polymerase (PARP) inhibitor. We further identify transcriptome expression of inhibitor of DNA biding 2 (ID2) as a potential predictive biomarker for treatment response to olaparib.

Conclusions: Together, our results demonstrate the potential utility of rapid drug screening combined with genomic profiling for precision treatment of gynecologic cancers.

Keywords: Gynecologic malignancy, Pharmacogenomic analysis, PARP inhibitor, TP53 mutations, ID2

\section{Background}

A fundamental principle of precision oncology is that molecular profiling of the tumor enables identification of appropriate therapeutic choice for individual patients [1-8]. However, predicting successful therapies on the sole basis of computational approach remains challenging [9-11]. Large-scale pharmacogenomic analyses using conventional

\footnotetext{
* Correspondence: jinkulee@ajou.ac.kr; nsnam@skku.edu;

garden.lee@samsung.com

†Jason K. Sa, Jae Ryoung Hwang and Young-Jae Cho contributed equally to this work.

${ }^{17}$ Department of Biochemistry and Molecular Biology, Ajou University School of Medicine, Suwon, Republic of Korea

${ }^{1}$ Institute for Refractory Cancer Research, Samsung Medical Center, Seoul, Republic of Korea

Full list of author information is available at the end of the article
}

cancer cell-line models have shown significant conceptual advances in discovering alternative therapeutic options for subsets of cancer patients [12-18]. However, molecular and pharmacological discrepancies between patient tumors and long-term cultured cancer cell-lines discourage clinical application of current gene-drug atlas. We have previously established a pharmacogenomic landscape of patientderived tumor cell (PDC) models to reveal unprecedented insights into dynamic gene-drug associations and demonstrated its clinical feasibility [19]. To further interrogate the dynamics of pharmacogenomic interactions at single tumor-lineage resolution, we generated a collection of gynecologic tumors, including cervical, endometrial/uterine, and epithelial ovarian cancers (EOCs), and explored 
potential gene-drug associations against 37 molecularly targeted agents.

Currently, there are over 100,000 newly diagnosed cases and approximately 32,000 mortalities from gynecologic cancers in the US. Gynecologic tumors can be categorized into 5 distinct subgroups: ovarian, endometrial/uterine, cervical, vulvar, and vaginal tumors based on geographical locations. The current standard treatment consists of aggressive surgical intervention followed by platinum-taxane chemotherapy. Despite such intensive treatment modalities, approximately $25 \%$ of the patients invariably undergo tumor relapse within 6 months from the initial treatment and there is no alternative therapeutic avenue that is readily available. Although large-scale genomic characterizations of ovarian, uterine, and cervical cancers have been profiled by The Cancer Genome Atlas (TCGA) Research Network [20-23], clinical application potential of molecular targeted therapy remains obscure. Toward this goal, we have established a library of short-term cultured PDC models and performed comprehensive analyses of pharmacogenomic interactions to identify potential molecular determinants that could guide personalized treatment in gynecologic tumors.

\section{Results}

\section{Establishment of patient-derived gynecologic tumor cell} library

To establish a gynecologic PDC library, we have collected 139 tumor specimens from patients who were diagnosed with either cervical (CC) $(n=18)$, uterine/endometrial $(n=$ $29)$, or epithelial ovarian $(n=92)$ cancers (Fig. 1a, Additional file 2: Table S1). Among them, 129 tumor tissues were subjected to targeted exome sequencing to identify genomic variations, including single nucleotide variants, short insertions and deletions, and copy number alterations $[19,24]$. Somatic variants were determined though exome sequencing (median of 20 genomic variants per sample), and only mutations with variant allele frequency of $>5 \%$ were considered. Whole-transcriptome sequencing (WTS) was performed on 51 tumors to curate gene expression profiles. The mutational landscape of gynecologic tumors revealed enrichment of TP53 somatic mutations in EOCs and endometrial cancers (EC) (Fig. 1b). BRCA1 or 2 mutations were observed in $35 \%, 53 \%$, and $38 \%$ of the sequenced tumors in ovarian, endometrial, and cervix cancers, respectively. Notably, genomic aberrations of Phosphoinositide 3-kinase (PI3K) pathway encoding genes including PIK3CA and PTEN were significantly more prevalent in endometrial tumors $\left(P=1.518 \times 10^{-06}\right.$ and $P=2.686 \times 10^{-08}$, Fisher's exact test; Additional file 1: Figure S1), suggesting potential therapeutic opportunities for PI3K targeted therapies [22, 25, 26]. Furthermore, somatic mutations of CTNNB1 were predominantly observed in ECs compared with other gynecologic cancer types
$\left(P=3.153 \times 10^{-03}\right.$, Fisher's exact test) [22], indicating that targeting of $\mathrm{Wnt} / \mathrm{B}$-catenin pathway could potentially provide clinical benefits for EC patients [27, 28]. Compared with TCGA datasets, our cohort constituted comparable levels of major cancer-driver genes in respective cancer types, including somatic mutations of TP53 in EOCs, PIK3CA and PTEN in cervical cancers, and PTEN, PIK3CA, ARID1A, and CTNNB1 in uterine corpus endometrial carcinomas (Additional file 1: Figure S2).

Tumor cell isolates were cultured under serum-free conditions for generally 2 to 4 weeks with epidermal growth factor (EGF) and basic fibroblast growth factor (FGF) supplements for enrichment of tumor initiating cell (TIC) populations (Additional file 2: Table S1) [29, 30]. Afterwards, PDCs were subjected to systematic drug sensitivity screening against 37 anti-cancer agents, targeting major oncogenic pathways including receptor tyrosine kinase (RTK), histone deacetylase (HDAC), and poly (ADP-ribose) polymerase (Additional file 3: Table S2 and Additional file 1: Figure S3) [19]. Drug sensitivities were determined using the area under curve (AUC) of the dose response curve (Fig. 1a) [19, 31-33]. A number of PDCs were further subjected to targeted exome sequencing and/or WTS to investigate whether the major gynecologic cancer-driver genes were retained from the parental tumors to PDCs. Consistent with previous observation, major drug-target genetic aberrations, including TP53, ERBB3, EGFR, and BRAF, were highly conserved in PDCs (Fig. 1c, Additional file 1: Figure S4). Moreover, transcriptome analysis of the parental tumors with matched PDCs demonstrated a strong positive correlation (Fig. 1c). To assess tumorigenicity of PDCs in vivo, we established patient-derived xenograft (PDX) models and evaluated their histological features [34]. Notably, PDX models recapitulated the original morphologic and pathologic characteristics of their parental tumors in situ (Additional file 1: Figure S5). Collectively, these results suggest that our gynecologic PDCs manifest molecular characteristics of the parental tumors and can be employed as surrogates for comprehensive pharmacogenomic analyses.

\section{Therapeutic landscape of gynecologic cancers reveals tumor type-specific drug sensitivity}

Next, we established a pharmacological landscape of 66 PDCs that were derived from cervical $(n=6)$, endometrial $(n=10)$, uterine $(n=8$, including leiomyoma), and EOCs ( $n=42)$ using 37 molecularly targeted drugs. A total of 2442 drug-PDC combinations were analyzed and plotted (Additional file 4: Table S3 and Additional file 1: Figure S6). Distribution of drug sensitivities varied widely, portraying the heterogeneous nature of gynecologic PDCs. A subset of drugs, including afatinib, dacomitinib, neratinib (EGFR), AZD2014 (mTOR), panobinostat (HDAC), and trametinib (MEK), showed exceptionally high anti-tumor activities 


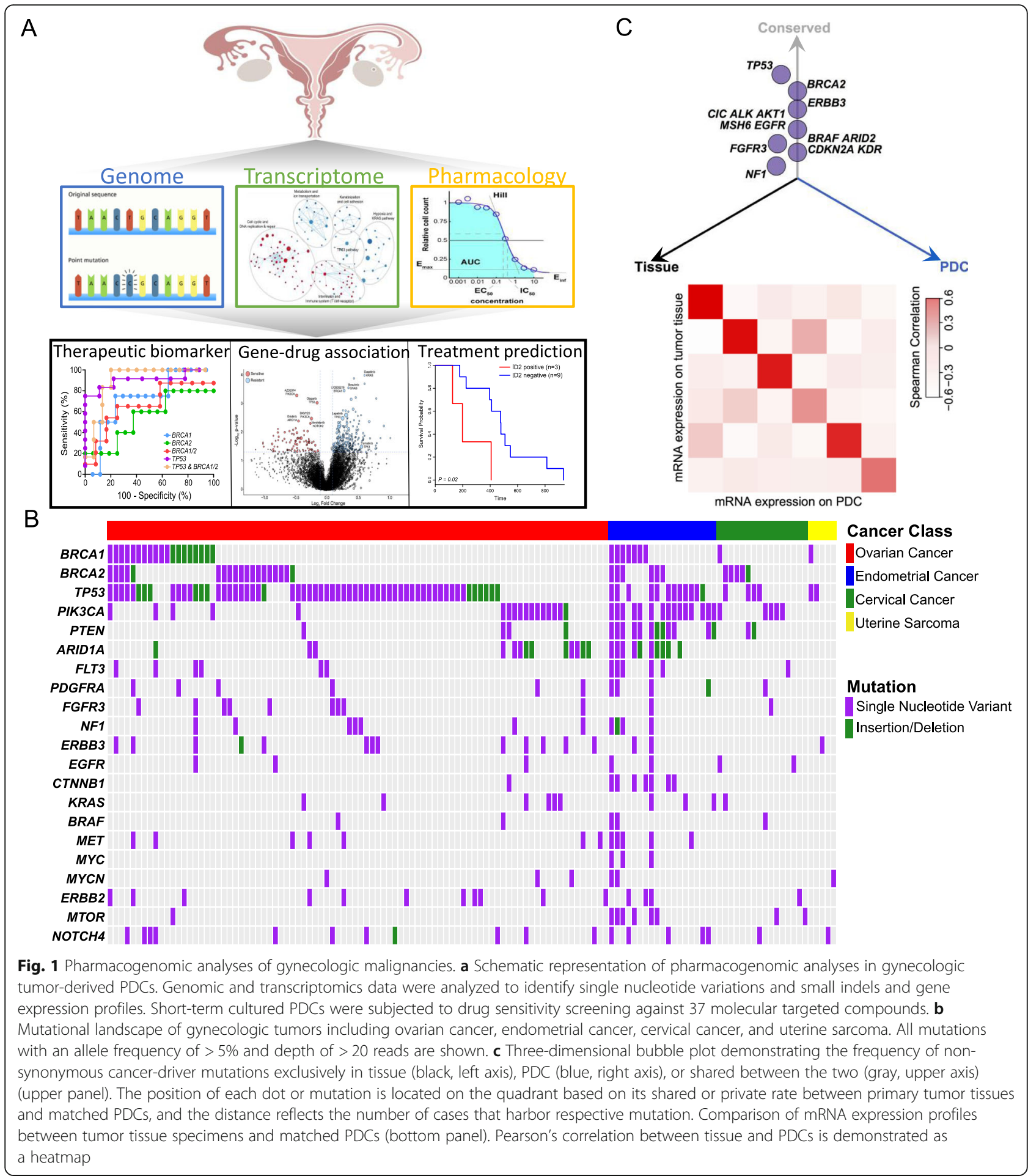

across all gynecologic tumors. In contrast, several agents, such as cabozantinib (VEGFR, MET, RET), ABT-888 (PARP), dabrafenib (BRAF), imatinib (Bcr/Abl), and sunitinib (PDGFR), demonstrated relatively minimal anti-cancer activities [19].

The molecular variations across diverse pathological tumor types could significantly contribute to distinct cancer type-specific drug response [12, 19]. Through lineage-specific drug sensitivity analysis (Additional file 5 : Table S4), we discovered that EGFR inhibitors including erlotinib, dacomitinib, and ibrutinib, a BTK inhibitor that was previously shown to have profound anti-EGFR activities $[19,35]$, were highly sensitive in EOCs $(P=$ $2.91 \times 10^{-02}, \quad P=3.59 \times 10^{-02}$, and $P=2.24 \times 10^{-02}$, 


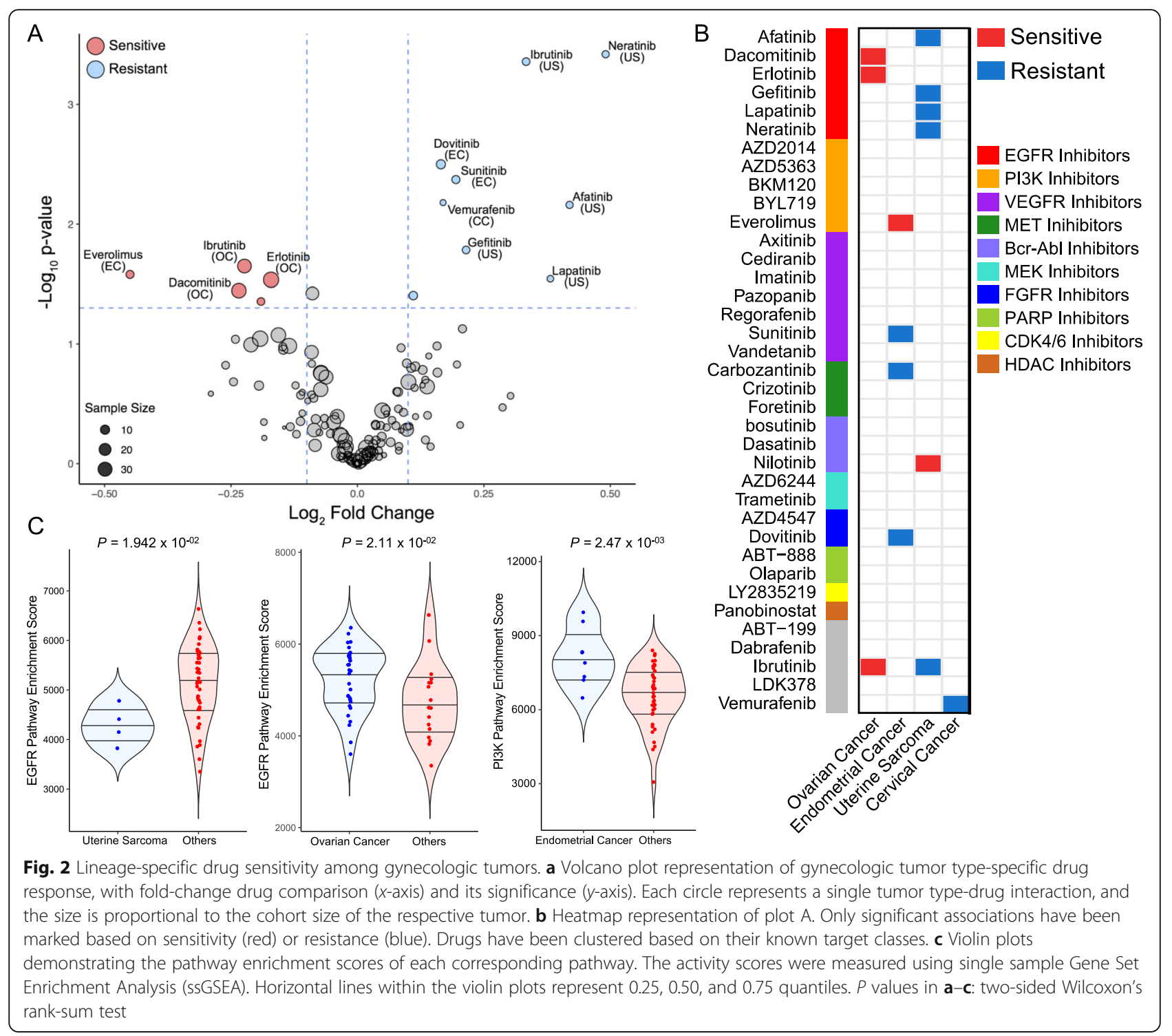

respectively; Wilcoxon's rank-sum test; Fig. 2a, b), whereas uterine sarcomas were considerably resistant to a number of EGFR-targeted compounds, including neratinib, afatinib, gefitinib, and ibrutinib $\left(P=3.81 \times 10^{-04}\right.$, $P=6.91 \times 10^{-03}, P=1.65 \times 10^{-02}$, and $P=4.39 \times 10^{-04}$, respectively; Wilcoxon's rank-sum test; Fig. $2 \mathrm{a}$, b). Consistently, pathway enrichment analysis showed activation and downregulation of EGFR-associated pathway in EOCs and uterine sarcomas, respectively (Fig. 2c). Furthermore, we also discovered enrichment of PI3K pathway in ECs, which further advocated our previous observation on recurrent genomic aberrations of PI3K-AKT-mTOR (PAM) pathway in ECs (Fig. 1b) [29]. Consistently, everolimus, a mTOR inhibitor, exhibited significantly higher anti-tumor activities in ECs compared with other cancer types (Fig. 2a, b) [36].

\section{Pharmacogenomic interactions in EOCs}

EOCs can be primarily categorized into two distinct subtypes based on their histopathological features: serous and clear cell carcinomas [37]. Since histologic characteristics largely contribute to diverse molecular and phenotypic states, we suspected that a wide-range of EOC-derived PDCs would demonstrate cell type-specific pharmacogenomic interactions. We first analyzed genomic profiles of EOCs using targeted exome sequencing (Fig. 3a and Additional file 6: Table S5). The mutational frequencies of $B R C A 1$ and BRCA2 were approximately 26 and $25 \%$, respectively, in serous subtype tumors. Conversely, only a small fraction of clear cell carcinomas harbored $B R C A 1$ or 2 mutations (8\% for both $B R C A 1$ and BRCA2; Fig. 3a). As previously reported, TP53 mutation was highly enriched in high-grade serous carcinomas (HGSC; 82\%), while only 


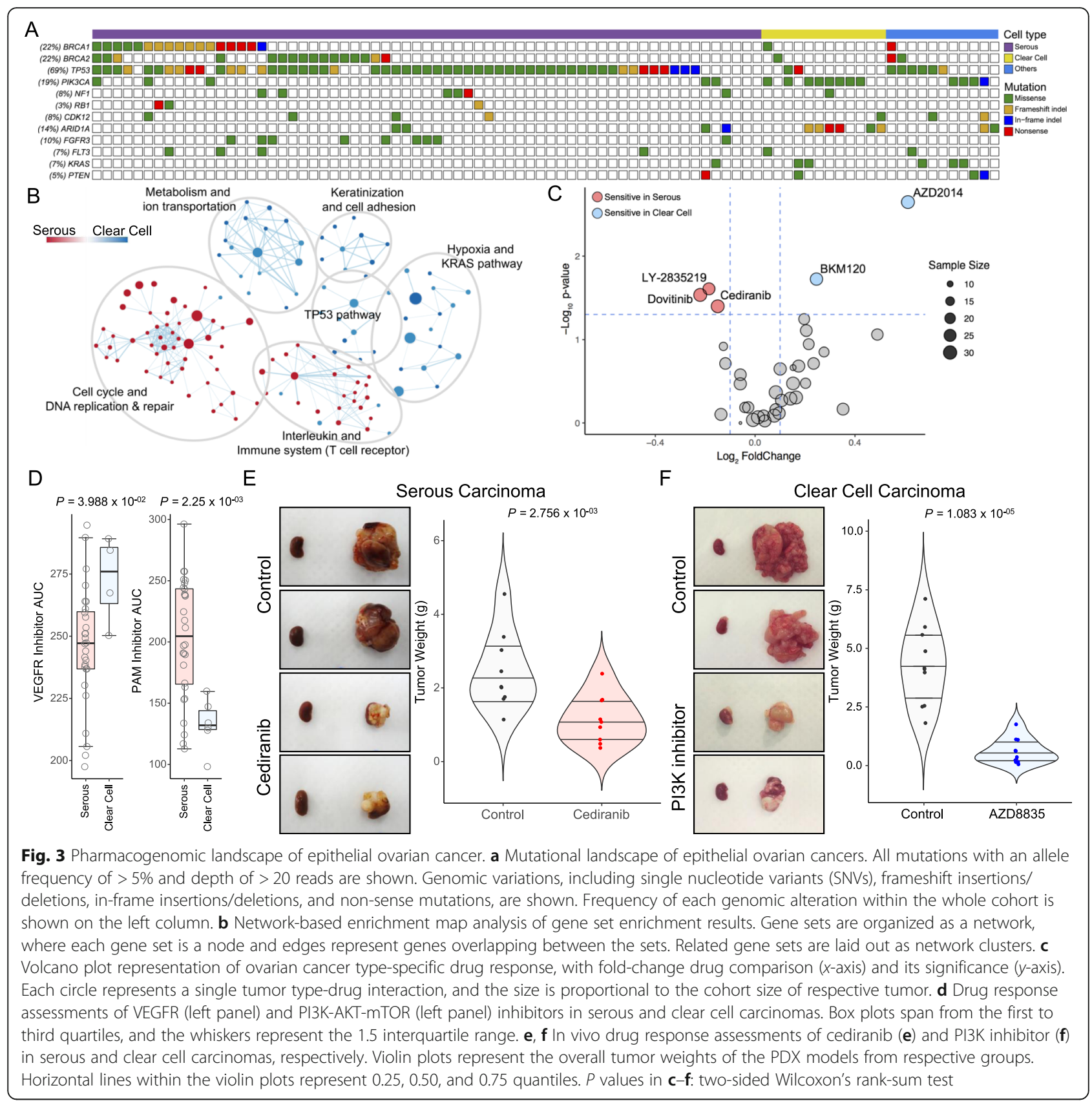

$17 \%$ of clear cell tumors harbored somatic mutation of TP53 $\left(P=2.179 \times 10^{-05}\right)[38,39]$. Notably, clear cell carcinomas were marked by high prevalence of PI3KCA and ARID1A mutations $(67 \%$ and $50 \%$ for PIK3CA and $A R I D 1 A$, respectively), compared to HGSC and other EOC types $\left(P=1.067 \times 10^{-05}\right.$ and $\left.P=5.43 \times 10^{-04}\right)[40-42]$. Surprisingly, other EOCs demonstrated considerably high levels of KRAS and PTEN genomic mutations (Additional file 1: Figure S7). To explore dynamic cellular signaling pathways that are enriched between HGSC and clear cell tumors, we conducted Gene Set Enrichment Analysis (GSEA) using gene expression profiles (Fig. 3b).
Interestingly, cell cycle and DNA replication/repair-associated pathways were significantly enriched in HGSC subtype. Additionally, interleukin and immune system encoding genes, including $\mathrm{T}$ cell receptor pathways, were profoundly more activated in HGSCs. In contrast, activations of metabolism/ion transport, keratinization/cell adhesion, hypoxia/KRAS, and TP53 signaling pathways were more predominant in clear cell tumors (Fig. 3b). Next, we examined differential drug sensitivity between HGSC and clear cell tumors to various molecularly targeted agents (Fig. 3c and Additional file 7: Table S6). Notably, several multi-kinase inhibitors, including dovitinib (FLT-3/c-Kit, 
FGFR1/3, and VEGFR) and cediranib (VEGFR, FIT1/4, and c-KIT), showed significantly high anti-tumor activities in HGSCs $\left(P=2.92 \times 10^{-02}\right.$ and $P=3.98 \times 10^{-03}$ for dovitinib and cediranib, respectively). HGSCs were also widely sensitive to LY-2835219, a CDK4/6 inhibitor $\left(P=2.46 \times 10^{-03}\right)$, as well. As inferred by enrichment of PIK3CA somatic mutation, clear cell carcinomas demonstrated superior sensitivities to PAM pathway inhibitors, including BKM120 (PI3K, $P=1.88 \times 10^{-03}$ ) and AZD2014 (mTOR, $P=2.25 \times$ $10^{-03}$ ). Comparative analysis between serous and clear cell carcinomas also revealed that serous type tumors were markedly sensitive to VEGFR inhibitors, including cediranib, pazopanib, and sunitinib (Fig. 3d). These findings were consistently confirmed in vivo using PDX models that were established from HGSC (Fig. 3e) and clear cell type PDCs (Fig. 3f).

\section{Identification of genomic biomarkers for drug sensitivity in gynecologic cancers}

Previous studies have shown that a single genetic alteration could be employed as a surrogate biomarker for predicting clinical response to various drug classes $[4,5$, $9,10,12,13,19]$. To identify genomic correlates of diverse pharmacological responses in gynecologic tumors, we evaluated individual drug profiles against single genomic alterations (Fig. 4a, Additional file 8: Table S7). Among the numerous interactions, PIK3CA mutation was the most robust genomic predictor of sensitivity towards PAM pathway inhibitors, including AZD2014 $\left(\mathrm{mTOR}, P=5.20 \times 10^{-04}\right)$ and BKM120 (PI3K, $P=3.34 \times$ $10^{-03}$ ). Pharmacological activities of erlotinib (EGFR) and vandetanib (VEGFR) were significantly associated with genomic aberrations of ARID1A $\left(P=4.07 \times 10^{-03}\right)$ and NOTCH2 $\left(P=4.96 \times 10^{-03}\right)$, respectively. BRCA1 mutation was also linked to therapeutic resistance against a CDK4/6 inhibitor, LY2835219 $(P=3.53 \times$ $\left.10^{-04}\right)$. Gynecologic PDCs that harbored somatic mutations in KRAS and GNAS were widely resistant to dasatinib (Bcr-Abl, $P=1.03 \times 10^{-04}$ ) and bosutinib (Abl and SRC, $P=2.81 \times 10^{-04}$ ), respectively. TP53-mutant tumors were highly resistant to a wide range of therapeutics, including lapatinib (EGFR, $P=2.52 \times 10^{-03}$ ), AZD5363 (AKT, $P=3.28 \times 10^{-03}$ ), and trametinib (MEK, $P=$ $\left.3.24 \times 10^{-02}\right)$. Conversely, TP53 mutated tumors were significantly sensitive to olaparib (PARP) treatment $(P=$ $\left.9.39 \times 10^{-04}\right)$.

PARP inhibition demonstrated potent therapeutic efficacies in patients who were diagnosed with either metastatic breast or advanced ovarian cancers with germline BRCA1/ 2 mutations [43, 44]. Although statistically not significant, BRCA1/2 mutations were enriched in olaparib-sensitive PDCs (represented with $Z$-score $<0$ ) (Fig. 4b). Interestingly, TP53 mutation also portrayed profound anti-tumor activity towards olaparib, regardless of histopathological subtype. Receiver operating characteristic (ROC) analysis revealed that genomic alterations of BRCA1/2 demonstrated positive correlations with olaparib sensitivity (AUC of ROC >0.5; Fig. 4c). Furthermore, sole TP53 mutation or combination of TP53 with BRCA1/2 mutations revealed enhanced predictability to olaparib treatment (AUC $=0.9074$ and 0.9 for TP53 and TP53 with BRCA1/2, respectively; Fig. 4c). To functionally validate our findings, we established cancer cell-line models that stably express various dominant-negative mutant forms of TP53 (R273H, R249S, and R175H) [45] in an OVISE ovarian cancer cell-line (TP53 wild-type). As suspected, cytotoxic activities of olaparib were significantly enhanced on all TP53 mutants $\left(\log \left(\mathrm{IC}_{50}\right)\right.$ values for TP53 wild-type, $\mathrm{R} 273 \mathrm{H}, \mathrm{R} 249 \mathrm{~S}$, and $\mathrm{R} 175 \mathrm{H}$ mutants were 2.155 (95\% CI 2.055 to 2.259 ), 1.421 (95\% CI 1.336 to 1.506 ), 1.269 (95\% CI 1.177 to 1.362 ), and 1.408 (95\% CI 1.296 to 1.520$) \mu \mathrm{M}$, respectively) (Fig. 4d).

\section{Transcriptomic correlates of olaparib response in EOCs}

Transcriptome analysis enables identification of unique gene-signature correlates for particular drug sensitivity $[12,13,19]$. To identify potential molecular determinants to olaparib response, we systematically analyzed the transcriptome profiles of EOCs based on their pharmacological responses to olaparib [44]. We discovered that expressions of SRC pathway encoding genes were significantly enriched in olaparib-resistant PDCs, while olaparib-sensitive tumors were marked by activation of NF-kB pathway (Fig. 5a). Functional validation of SRC inhibition showed that saracatinib (SRC, $20 \mu \mathrm{m}$ ) significantly augmented the therapeutic effects of olaparib in BRCA1-mutant PDCs $\left(P=3.73 \times 10^{-04}\right.$; Fig. 5b). Among the list of enriched SRC pathway-encoding genes, mRNA expressions of ID1/2/3 were predominantly upregulated in olaparib-resistant PDCs (Fig. 5c). Especially, transcriptome expression level of ID2 was significantly correlated with therapeutic resistance to olaparib treatment $(r=0.52, P=0.01978$; Fig. $5 d)$. To determine whether ID2 expression could be employed as a potential predictor of response to olaparib, we retrospectively analyzed treatment-free survival of 41 patients (ID2 positive, $n=17$; ID2 negative, $n=24$ ), who were previously diagnosed with HGSC, harbored BRCA1 or 2 mutation, and received olaparib treatment. Notably, the Kaplan-Meier survival analysis revealed that patients with low ID2 expression demonstrated significantly prolonged treatment-free survival to olaparib (log rank test, $P=0.02$, median survival: ID2 ${ }^{\text {pos }} 4.03$ months vs. ID2 ${ }^{\text {neg }} 8.73$ months; 95\% CI: ID2 ${ }^{\text {pos }}$ 2.87 6.67 vs. ID2 ${ }^{\text {neg }} 5.60 \sim 13.87$; Fig. 5e, f). Collectively, our results indicate that enrichments of SRC pathway and ID2 expression relate to therapeutic resistance to olaparib treatment in EOCs. 


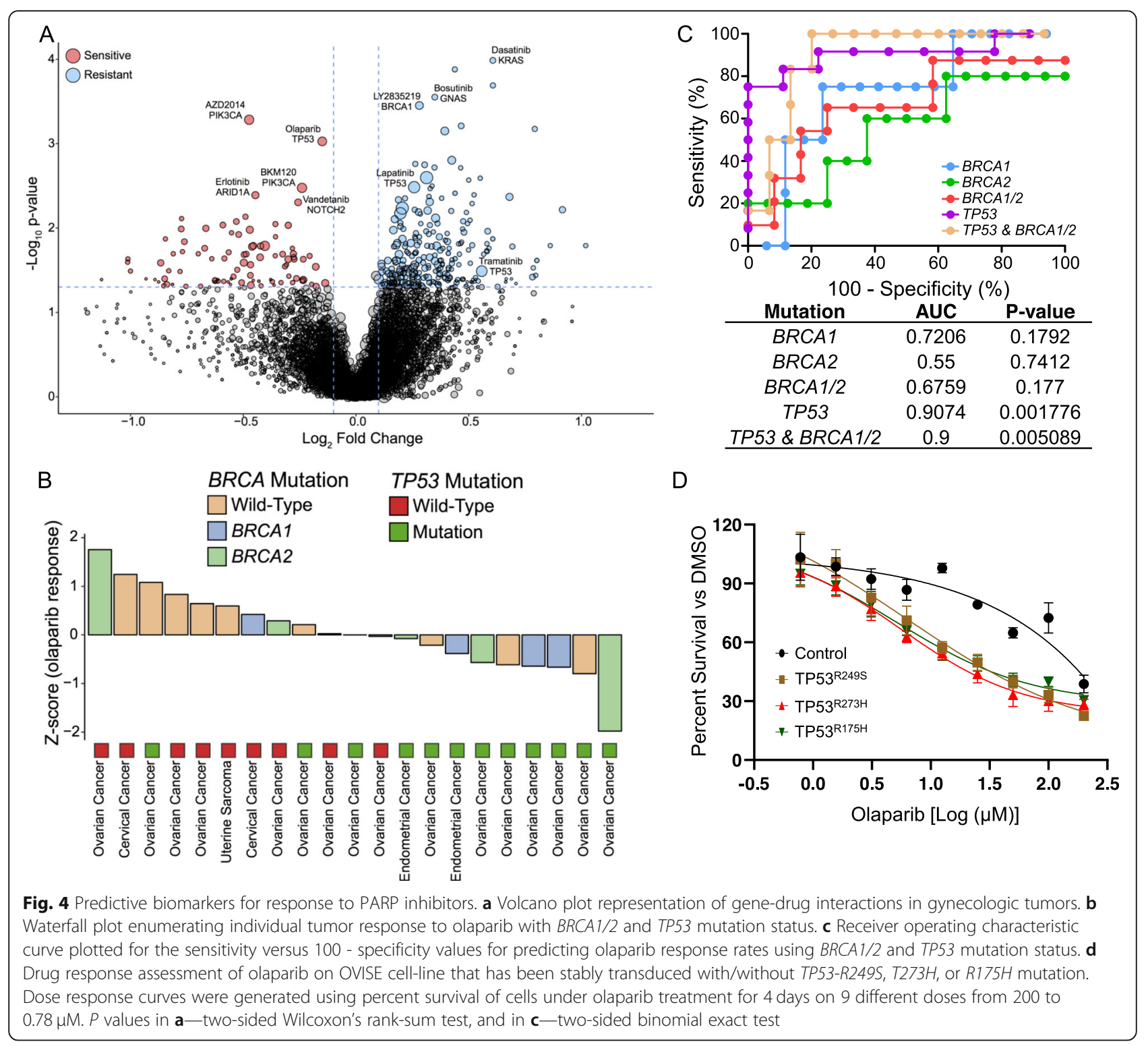

\section{Discussion}

The success of precision oncology depends on identification of effective drugs tailored to individual patients based on molecular profiling of the tumor. Comprehensive analyses of cancer genome have revealed a landscape of key genetic ablations that constitute complex process of tumorigenesis $[1,8,46]$. A large-scale compilation of pharmacological drug response with molecular characterization of cancer cell-line models has provided a reference point for evaluating potential genomic markers of drug sensitivity [12, 13, 16, 17]. Moreover, we have previously established a landscape of pharmacogenomic interactions using a library of short-term cultured PDCs to explore dynamic gene-drug associations and presented its clinical feasibility [19]. As an extension, we generated a collection of 139 gynecologic tumors from patients with cervical, endometrial/uterine, or ovarian cancers. Through integrative genomic, transcriptomic, and pharmacological analyses, we have provided several new therapeutic insights for gynecologic malignancies (Fig. 1a).

We evaluated lineage-specific drug sensitivity in gynecologic tumors and discovered that EOCs demonstrated enhanced sensitivities to multiple EGFR inhibitors, while ECs were particularly sensitive to everolimus, an mTOR inhibitor. A number of clinical observations further advocated our results. Despite the small number of patients and lack of randomization, addition of erlotinib (EGFR) to platinum or paclitaxel provided favorable clinical outcomes for EOC patients, compared to platinum or paclitaxel treatment alone [47]. Moreover, several clinical investigations demonstrated potential therapeutic benefits of mTOR 

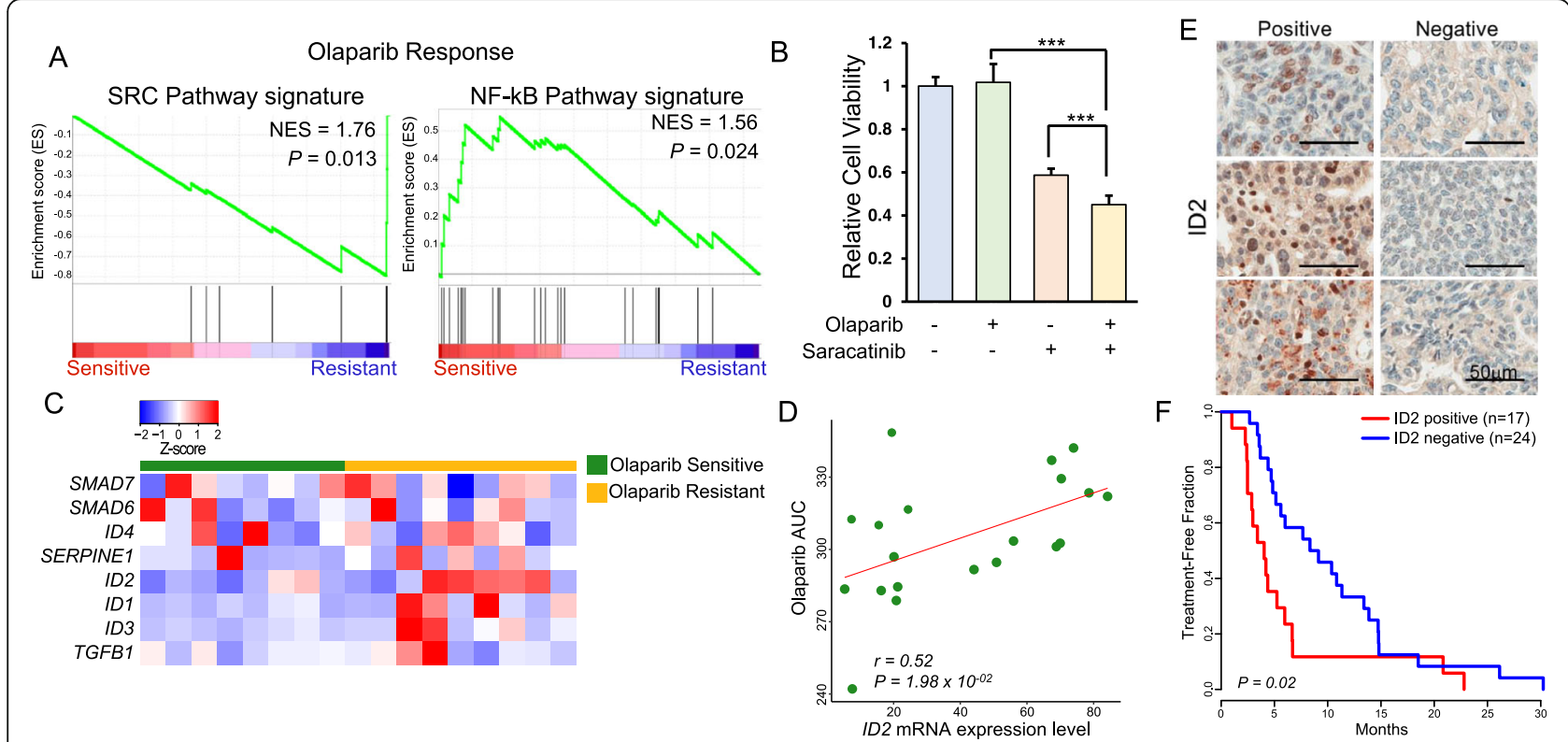

Fig. 5 Transcriptomic correlates of olaparib sensitivity. a Gene Set Enrichment Analysis (GSEA) between olaparib-sensitive and olaparib-resistant PDCs. $\mathbf{b}$ Drug response assessment of olaparib and/or saracatinib. $\mathbf{c}$ Heatmap representation of SRC pathway encoding gene expressions in olaparib-sensitive and olaparib-resistant PDCs. d A scatter plot demonstrating linear correlation between olaparib AUC and ID2 expression. The correlation coefficient and the $P$ value were obtained using Pearson's correlation test. e Representative immunohistochemical images of ID2 staining in patient tumor specimens. Scale bars, $50 \mu \mathrm{m}$. $\mathbf{f}$ The Kaplan-Meier treatment-free survival analysis of patients with high vs. low ID2 protein expression levels. $P$ values in $\mathbf{b}$ - two-tailed $t$ test, in $\mathbf{d}$-Pearson's correlation test, and in $\mathbf{f}$-log-rank test

targeted therapy in EC patients [36, 48-50]. Consistent with previous observations, we confirmed that serous and clear cell EOCs can be distinguished by evidently distinct genomic compositions (Fig. 3a), highlighting the need for molecular-based therapeutic approaches. Interestingly, our drug screening results, coupled with in vivo validations, propose clinical utility of cediranib (VEGFR) for HGSC patients, while PAM inhibitors could be more beneficial for those with clear cell carcinomas. Recent phase II and III clinical trials with cediranib also revealed that cediranib plus olaparib treatment resulted in a significant improvement in progression-free survival of HGSC patients compared to olaparib alone [51, 52]. As in vivo PDX results were only representative cases, further functional validations in a larger cohort are warranted to explore potential clinical applications of VEGFR and PAM inhibitors in serous and clear cell type tumors, respectively.

We also identified genomic correlates of drug sensitivity and resistance to olaparib. Approximately $13-18 \%$ of the HGSCs are attributable to BRCA1 or 2 germline mutations, and PARP inhibition therapy has been a successful approach for these patients. However, the need for discovering an alternative molecular biomarker to better predict the clinical efficacy against PARP inhibition treatment has been increasingly recognized due to global acquisition of olaparib resistance. Notably, we demonstrated that addition of TP53 mutation could be a significant molecular determinant for predicting potential clinical response to PARP inhibition therapy. The tumor suppressor protein $\mathrm{p} 53$ provides an essential role in governing cell cycle arrest or apoptosis upon DNA damage [53]. However, the underlying molecular cascades that determine $\mathrm{p} 53$ protein stability and its activation are not fully understood. Accumulation of PARP1 is an early event where a single strand DNA break is generated and initiates base excision repair (BER) pathway [54]. PARP-1 interacts with p53 to modulate DNA damage [55], and genotoxic drugs promote accumulation and activation of p53 in parp-1-deficient cells. Furthermore, p53 deficiency enhanced pharmacological sensitivity towards PARP inhibition therapy in mantle cell lymphoma [55]. Consistent with such findings, our results suggest the prospect of targeting p53-deficient tumors with PARP inhibitors regardless of histopathological characteristics. Prevalence of TP53 mutation in HGSCs could contribute to the clinical success of PARP inhibitors [39, 42, 44].

Lastly, we identified SRC activation to be associated with therapeutic resistance to olaparib. The SRC family of non-receptor tyrosine kinases regulates essential cellular programs, including cellular proliferation, differentiation, migration, survival, and angiogenesis [56]. A substantial number of studies have postulated that activation of SRC pathway contributes to inherent resistance to chemotherapy and inhibition of SRC pathway could potentially circumvent such mechanism [57, 58]. Moreover, transcriptional expressions of ID family genes were identified as molecular correlates of olaparib sensitivity. 
ID proteins are members of the large family of the helixloop-helix (HLH) transcription factors. During development, ID proteins govern cell cycle and differentiation programs by modulating various cell cycle regulators [59]. From the perspective of tumorigenesis, upregulation of ID protein is mediated by a group of protooncogenes, including $M y c$, Ras, and (EWS)-Ets, and prevents activation of various tumor suppressor genes [60], making it a promising therapeutic target [61].

\section{Conclusion}

Here, we generated an additional cohort at single tumorlineage resolution, specializing in gynecologic malignancies. We performed systematic analyses of tumor genome and transcriptome to identify molecular determinants that dictate drug sensitivity to various molecular targeted drugs that are currently being used or under development. Our work provides an extension to current pharmacogenomic database in identifying predictive biomarkers and combinational approach to overcome cellular-intrinsic resistance to particular drug classes, including PARP inhibitors.

\section{Methods}

Gynecologic cancer specimens and their derivative cells

After receiving informed consent, tumor specimens and clinical records were obtained from patients undergoing surgery at Samsung Medical Center (SMC) in accordance with its Institutional Review Board. Surgical samples measuring $\sim 5 \times 5 \times 5 \mathrm{~mm}^{3}$ were snap frozen using liquid nitrogen for genomic analysis. Portions of the surgical samples were enzymatically dissociated using Liberase TM (Roche) and cultured in DMEM/F12 media with L-glutamine (Thermofisher), N2 and B27 supplements (0.5× each; Thermofisher), human recombinant basic fibroblast growth factor (bFGF), and epidermal growth factor (EGF; $20 \mathrm{ng} / \mathrm{ml}$ each; $R \& D$ Systems).

\section{Orthotopic xenograft animal models and drug treatment} Female BALB/c nude mice were purchased from ORIENT BIO (Sungnam, Korea). This study was performed in accordance with relevant guidelines and regulations. This study was reviewed and approved by the Institutional Animal Care and Use Committee (IACUC) of Samsung Biomedical Research Institute (SBRI). SBRI is an Association for Assessment and Accreditation of Laboratory Animal Care International (AAALAC International, protocol no. H-A9-003)-accredited facility and abides by the Institute of Laboratory Animal Resources (ILAR) guidelines. To generate PDX models, patient tumor specimens were cut into small pieces (below 2-3 mm), implanted into the subrenal capsule of the left mouse kidney, and propagated by serial transplantation [34]. After 1-2 weeks, the mice ( $n=$ 10 mice per group) were treated with either $1 \%$ polysorbate 80 or $0.5 \%$ methylcellulose or cediranib (6 $\mathrm{mg} / \mathrm{kg}$, qd, p.o.) or AZD8835 (25 mg/kg, bid, p.o.). Mice were monitored daily for tumor development and postoperative complications and were sacrificed between day 35 and 40 or if mice seemed moribund. Total body weight and tumor weight of each mouse were recorded. Tumors were fixed in formalin and embedded in paraffin or snap frozen in the OCT compound (Sakura Finetek, Japan, Tokyo, Japan) in liquid nitrogen.

\section{Isolation of genomic DNA and quality control}

Genomic DNA was extracted from fresh tissue specimens using the QIAamp DNA mini kit (Qiagen, Valencia, CA, USA) or from FFPE tissues using either the Promega Maxwell 16 CSC DNA FFPE kit or the QIAamp DNA FFPE Tissue kit according to the manufacturer's manual. The purity, amount, and median size of the extracted DNA were measured by the Nanodrop 8000 UV-Vis spectrometer (Thermo Scientific Inc., Wilmington, DE, USA), Qubit 2.0 fluorometer (Life Technologies Inc., Grand Island, NY, USA), and 2200 TapeStation Instrument (Agilent Technologies, Santa Clara, CA, USA). In addition, $\triangle \mathrm{Ct}$ values were determined using real-time PCR (Agilent Technologies) with Mx3005p instrument (Agilent Technologies, USA), FFPE QC kit (Illumina, cat no. WG-3211001), and Brilliant Ultra-Fast SYBR Green qPCR (Agilent Technologies, cat no. 600882). If DNA meets the quality criteria such as (i) purity to absorption ratio $(260 \mathrm{~nm} / 280$ $\mathrm{nm})>1.8,260 \mathrm{~nm} / 230 \mathrm{~nm}>1.8$; (ii) total amount $>250 \mathrm{ng}$; (iii) degradation to $\Delta \mathrm{Ct}$ value $<2.0$; or DNA median size $>0.35 \mathrm{~kb}$, it is proceeded onto the sequencing step.

\section{Panel design and sequencing}

Samples were profiled on CancerSCAN, a targeted sequencing platform designed at Samsung Medical Center. This customized platform offers flexibility to include target genes that are curated from the literature or requested by researchers and clinicians. To obtain cancer panel sequencing data, CancerSCAN probes were designed to enrich the exons of 80 target genes (Additional file 9: Table S8). Genomic DNA was sheared using the Covaris S220 (Covaris, Woburn, MA) to construct a sequencing library using the SureSelect XT Reagent Kit, HSQ (Agilent Technologies) on target genes. A paired-end sequencing library was purified and amplified with a barcode tag, and the library quality and quantity were determined. Sequencing was carried out using the 100-bp paired-end mode of the TruSeq Rapid PE Cluster kit and TruSeq Rapid SBS kit on a HiSeq 2500 sequencing platform (Illumina, San Diego, CA, USA).

\section{Bulk RNA sequencing}

RNA-seq libraries were prepared using the Illumina TrueSeq RNA Sample Prep kit. Sequenced reads were 
mapped onto hg19 using the Burrows-Wheeler Aligner (BWA). The initial alignment BAM files were sorted and summarized into BED files using SAMtools and bedTools. The BED files were used to calculate values of RPKM (reads per kilobase of transcript per million reads) for each gene, using DEGseq package.

\section{Drug screening}

PDCs were cultured in serum-free medium, dissociated into single cells, and seeded in 384-well plates at a density of 500 cells per well in duplicate or triplicate for each treatment. The drug panel consisted of 37 anti-cancer agents targeting oncogenic signals. All drug libraries were purchased from Selleckchem. PDCs were treated with drugs in a four-fold and seven-point serial dilution series from 20 to $4.88 \mathrm{nM}$ using a Janus Automated Workstation (PerkinElmer, Waltham, MA, USA). After 7 days of incubation at $37^{\circ} \mathrm{C}$ in a $5 \% \mathrm{CO}_{2}$ humidified incubator, cell viability was analyzed using an adenosine triphosphate (ATP) monitoring system based on firefly luciferase (ATPLite $^{\mathrm{mx}}$ 1step, PerkinElmer). Viable cells were estimated using an EnVision Multilabel Reader (PerkinElmer). The controls, dimethyl sulfoxide (DMSO) vehicle, were used to calculate relative cell viability for each plate and to normalize the data on a per-plate basis. Dose response curve (DRC) fitting was performed using GraphPad Prism 5 (GraphPad) and evaluated by measuring the area under curve (AUC). In brief, each plate was normalized to the mean of the seven conditions on the plate with a DMSO control. After normalization, best-fit lines and the resulting $\mathrm{IC}_{50}$ values were calculated using GraphPad: [log(inhibitor) vs. response variable slope (four parameters)]. $Y=$ Bottom + (Top - Bottom $) /\left(1+10^{\wedge}\left(\left(\log \mathrm{IC}_{50}-X\right) \times\right.\right.$ HillSlope $\left.)\right)$. The AUC of each curve was determined using GraphPad Prism, ignoring regions defined by fewer than two peaks. Non-convergence or ambiguous curves are excluded in every analysis.

\section{Pharmacogenomic interactions on major genomic alterations}

For gene-drug associations, a list of major cancer-driver alterations, including single nucleotide variations, small insertions, and deletions, was considered as a predictive genomic biomarker to evaluate drug response interactions. For each drug candidate, drug sensitivity data (AUCs) were analyzed by comparing tumors with the selected genomic alteration to those without using the Wilcoxon rank-sum test. Samples with unknown status of a given alteration were excluded from the analysis. To evaluate lineage-specific drug sensitivities in gynecologic tumors, drug sensitivity data were analyzed by comparing tumors from each pathologic entity to the rest using the Wilcoxon rank-sum test. For transcriptome analysis, tumors were classified as "sensitive" $(Z$-score $<-0.5)$ or "resistant" (Z-score $>0.5)$ based on drug sensitivity data and subjected to Gene Set Enrichment Analysis (GSEA).

\section{Plasmid preparation and stable cell establishment}

Lentiviral vectors encoding TP53 mutants (R175H, $\mathrm{R} 273 \mathrm{H}$, or R249S) in pLenti6/V5 plasmid were purchased from Addgene (cat no. 22936, 22934, and 22935, respectively). Lentivirus was prepared by transfecting plasmids into the 293T cells using pMD2G, psPAX2 (Addgene), and Lipofectamine 2000 (Thermofisher). After the initial transfection, supernatants were collected after 48 and $72 \mathrm{~h}$ and filtered through $0.45 \mu \mathrm{M}$ filters (Milipore). To generate stable TP53 mutant-expressing cell lines, lentivirus particles were incubated with ovarian clear cell carcinoma, OVISE, and treated with polybrene $(8 \mu \mathrm{g} / \mathrm{ml}$, Sigma) for $48 \mathrm{~h}$ and blasticidin $(5 \mu \mathrm{g} / \mathrm{ml}$, Sigma) selection was performed for 2 weeks.

\section{Immunohistochemistry}

Immunohistochemical staining was performed on formalin-fixed, paraffin-embedded, 4-5- $\mu \mathrm{m}$-thick tissue sections, using the Bond-maxTM automated immunostainer (Leica Biosystems, Melbourne, Australia) and BondTM Polymer Refines Detection Kit (Vision Biosystems, Melbourne, Australia). Mouse monoclonal anti-ID2 antibody (1:100; NBP2-66898, Novus Biologicals, USA) was used. Briefly, antigen retrieval was carried out at $97^{\circ} \mathrm{C}$ for $20 \mathrm{~min}$ in ER1 buffer. After blocking the endogenous peroxidase activity with $3 \%$ hydrogen peroxidase for $10 \mathrm{~min}$, primary antibody was incubated for $60 \mathrm{~min}$ at room temperature. To verify antibody specificity, antimouse IgG (AI-2000; Vector Laboratories, Burlingame, CA, USA) was used as a control. The degree of immunostaining of ID2 was evaluated according to staining proportion of positively stained cancer cell nucleus and the staining intensity, as previously described [62]. Briefly, the areas of stained cancer cells were scored as follows: the percentage of positive cells $(0-100 \%)$ and intensity scaled from 0 to 2 (null $=0$, weak to moderate $=1$, strong $=2$ ).

\section{Statistics}

All statistical analyses were conducted by either Wilcoxon's rank-sum test (two-sided), Pearson's correlation coefficient test, or Fisher's exact test (two-sided). Survival curves were estimated with the Kaplan-Meier method. All statistical analyses were conducted and obtained using the R software (https://www.r-project.org).

\section{Supplementary information}

Supplementary information accompanies this paper at https://doi.org/10. 1186/s13059-019-1848-3.

Additional file 1: Figure S1. Frequency of major cancer-driver gene alterations in gynecologic tumors. Figure S2. Frequency of major cancer- 
driver genes in SMC and TCGA cohorts. Figure S3. Drug panel classification. Figure S4. Preservation of copy number alterations in primary tissues and PDCs. Figure S5. Representation of histopathologic

characteristics between parental and patient-derived xenograft tumors. Figure S6. Pharmacological landscape of 37 molecular-targeted agents. Figure S7. Frequency of major cancer-driver gene alterations in ovarian cancer.

Additional file 2: Table S1. Descriptive characteristics of 139 samples from gynecologic cancers used in the drug screening and/or the genomic evaluations.

Additional file 3: Table S2. A list of the 37 drugs used in drug sensitivity analysis. The drugs were described by the chemical and/or generic names, their respective targets and clinical phases.

Additional file 4: Table S3. Area under the curve (AUC) values of 37 drugs in 66 PDCs. NA represents the AUC value extracted from non-fitted dose response curve (DRC) that resulted in a non-convergent or ambiguous curve.

Additional file 5: Table S4. Tumor type-specific drug associations identified using 37-drug library. Wilcoxon rank sum test was applied to determine the relative differences of drug sensitivity between certain tumor type and all the other cancers.

Additional file 6: Table S5. The genomic profile of gynecologic tumor samples which was identified using CancerSCANTM sequencing and analysis protocol

Additional file 7: Table S6. Cell type-specific drug associations in EOCs. Wilcox rank sum test was applied to determine the relative differences of drug sensitivity between serous and clear cell type tumors.

Additional file 8: Table S7. Pharmacogenomic associations identified using integrative analysis of drug sensitivity results (AUC) and genomic alteration. The statistical significance was calculated using Wilcoxon rank sum test.

Additional file 9: Table S8. List of genes $(n=80)$ and sequencing protocol for CancerSCANTM, detecting cancer-driven variant.

Additional file 10. Review History.

\section{Acknowledgements}

We thank the Samsung Medical Center BioBank for providing the biospecimens that were used in this study.

\section{Review history}

The review history is available as Additional file 10

\section{Authors' contributions}

JKS, JRH, and YJC are the co-first authors. JKS, JRH, and YJC performed the majority of the experiments and analyses. JWL, DHN, and JKL provided the concept of the study. JYR and JJC participated in several experiments. JJC, SYJ, JK, MSK, ESP, YYL, CHC, TJK, BGK, and DSB provided the surgical samples and performed the clinical interpretation. JSB and WYP generated the genome and transcriptome data. JKS, HJC, and HK processed and analyzed the sequencing data. YL, NGH, JWO, YJS, JYK, and YJS generated the patientderived cell library and conducted the drug response analysis. TL, HSK, SYS, $\mathrm{HDH}$, and HJA performed the pathological analysis of biospecimens. JKS, JKL, and JWL wrote the manuscript and organized the figures and tables. AKS and RR reviewed and edited the manuscript. JWL, DHN, and JKL designed and supervised the entire project. All authors read and approved the final manuscript.

\section{Funding}

This research was supported in part by a grant from the Korea Health Technology R\&D project through the Korea Health Industry Development Institute (KHIDI), funded by the Ministry of Health and Welfare, Republic of Korea (HI14C3418 and HI18C1953); the National R\&D Program for Cancer Control, Ministry for Health, Welfare and Family Affairs, Republic of Korea (1520100); and the National Research Foundation of Korea (NRF) Grant funded by the Korean Government (NRF-MSIP-2016R1A5A2945889, NRFMEST-2016R1A2B3006644, NRF-2017R1A2B4003434, and NRF2018R1C1B3001648)

\section{Availability of data and materials}

All sequenced data have been deposited in the European Genomephenome Archive (EGA) under accession EGAS00001003556 [63].

\section{Ethics approval and consent to participate}

The study was approved by the local committee on the use of human samples for experimental studies of the Samsung Medical Center (SMC), Seoul, Republic of Korea (IRB file \#201004004). Written informed consents were provided by the participants prior to enrollment. All experimental methods abided by the Helsinki Declaration.

\section{Consent for publication}

Not applicable

\section{Competing interests}

Do-Hyun Nam is the CEO of AimedBio Inc. and owns shares of AimedBio Inc. which owns IPs for Avatascan. The other authors declare that they have no competing interests.

\section{Author details}

${ }^{1}$ Institute for Refractory Cancer Research, Samsung Medical Center, Seoul, Republic of Korea. ${ }^{2}$ Research Institute for Future Medicine, Samsung Medical Center, Seoul, Republic of Korea. ${ }^{3}$ Department of Biomedical Sciences, Korea University College of Medicine, Seoul, Republic of Korea. ${ }^{4}$ Samsung Biomedical Research Institute, Samsung Medical Center, Sungkyunkwan University School of Medicine, Seoul, Republic of Korea. ${ }^{5}$ Department of Obstetrics and Gynecology, Samsung Medical Center, Sungkyunkwan University School of Medicine, Seoul, Republic of Korea. ${ }^{6}$ Department of Obstetrics and Gynecology, Dankook University Hospital, Cheonan, Republic of Korea. ${ }^{7}$ Department of Neurosurgery, Samsung Medical Center, Sungkyunkwan University School of Medicine, Seoul, Republic of Korea. ${ }^{8}$ Department of Health Sciences and Technology, Samsung Advanced Institute for Health Sciences \& Technology, Sungkyunkwan University, Seoul, Republic of Korea. ${ }^{9}$ Department of Pathology, Hwasun Hospital, Chonnam National University Medical School, Gwangju, Republic of Korea.

${ }^{10}$ Department of Pathology and Translational Genomics, Samsung Medical Center, Sungkyunkwan University School of Medicine, Seoul, Republic of Korea. " Samsung Genome Institute, Samsung Medical Center, Seoul, Republic of Korea. ${ }^{12}$ Department of Immunology, School of Medicine, Konkuk University, Chungju, Republic of Korea. ${ }^{13}$ Biomedical Research Institute, Korea Institute of Science and Technology, Seoul, Republic of Korea. ${ }^{14}$ Department of Gynecologic Oncology and Department of Cancer Biology, The University of Texas MD Anderson Cancer Center, Houston, TX, USA. ${ }^{15}$ Department of Systems Biology, Columbia University, New York, NY, USA. ${ }^{16}$ Department of Biomedical Informatics, Columbia University, New York, NY, USA. ${ }^{17}$ Department of Biochemistry and Molecular Biology, Ajou University School of Medicine, Suwon, Republic of Korea.

Received: 9 April 2019 Accepted: 2 October 2019

Published online: 26 November 2019

\section{References}

1. Brennan CW, Verhaak RG, McKenna A, Campos B, Noushmehr H, Salama SR, Zheng S, Chakravarty D, Sanborn JZ, Berman SH, et al. The somatic genomic landscape of glioblastoma. Cell. 2013;155:462-77.

2. Cancer Genome Atlas Research N, Weinstein JN, Collisson EA, Mills GB, Shaw KR, Ozenberger BA, Ellrott K, Shmulevich I, Sander C, Stuart JM. The Cancer Genome Atlas Pan-Cancer analysis project. Nat Genet. 2013;45:1113-20.

3. Hamburg MA, Collins FS. The path to personalized medicine. N Engl J Med. 2010;363:301-4.

4. Slamon DJ, Leyland-Jones B, Shak S, Fuchs H, Paton V, Bajamonde A, Fleming T, Eiermann W, Wolter J, Pegram M, et al. Use of chemotherapy plus a monoclonal antibody against HER2 for metastatic breast cancer that overexpresses HER2. N Engl J Med. 2001;344:783-92.

5. Chapman PB, Hauschild A, Robert C, Haanen JB, Ascierto P, Larkin J, Dummer R, Garbe C, Testori A, Maio M, et al. Improved survival with vemurafenib in melanoma with BRAF V600E mutation. N Engl J Med. 2011; 364:2507-16.

6. O'Brien SG, Guilhot F, Larson RA, Gathmann I, Baccarani M, Cervantes F, Cornelissen JJ, Fischer T, Hochhaus A, Hughes T, et al. Imatinib compared 
with interferon and low-dose cytarabine for newly diagnosed chronic-phase chronic myeloid leukemia. N Engl J Med. 2003;348:994-1004.

7. Loeb LA. Human cancers express mutator phenotypes: origin, consequences and targeting. Nat Rev Cancer. 2011;11:450-7.

8. Vogelstein B, Papadopoulos N, Velculescu VE, Zhou S, Diaz LA Jr, Kinzler KW. Cancer genome landscapes. Science. 2013;339:1546-58.

9. Rubio-Perez C, Tamborero D, Schroeder MP, Antolin AA, Deu-Pons J, PerezLlamas C, Mestres J, Gonzalez-Perez A, Lopez-Bigas N. In silico prescription of anticancer drugs to cohorts of 28 tumor types reveals targeting opportunities. Cancer Cell. 2015;27:382-96.

10. Altman RB. Predicting cancer drug response: advancing the DREAM. Cancer Discov. 2015;5:237-8.

11. Geeleher P, Cox NJ, Huang RS. Clinical drug response can be predicted using baseline gene expression levels and in vitro drug sensitivity in cell lines. Genome Biol. 2014;15:R47.

12. Garnett MJ, Edelman EJ, Heidorn SJ, Greenman CD, Dastur A, Lau KW, Greninger P, Thompson IR, Luo X, Soares J, et al. Systematic identification of genomic markers of drug sensitivity in cancer cells. Nature. 2012;483:570-5.

13. Barretina J, Caponigro G, Stransky N, Venkatesan K, Margolin AA, Kim S, Wilson CJ, Lehar J, Kryukov GV, Sonkin D, et al. The Cancer Cell Line Encyclopedia enables predictive modelling of anticancer drug sensitivity. Nature. 2012:483:603-7.

14. Shoemaker $\mathrm{RH}$. The $\mathrm{NCl} 60$ human tumour cell line anticancer drug screen. Nat Rev Cancer. 2006;6:813-23.

15. Basu A, Bodycombe NE, Cheah JH, Price EV, Liu K, Schaefer Gl, Ebright RY, Stewart ML, Ito D, Wang $S$, et al. An interactive resource to identify cancer genetic and lineage dependencies targeted by small molecules. Cell. 2013; 154:1151-61.

16. Holbeck SL, Collins JM, Doroshow JH. Analysis of Food and Drug Administration-approved anticancer agents in the NCl60 panel of human tumor cell lines. Mol Cancer Ther. 2010;9:1451-60.

17. Garnett MJ, McDermott U. The evolving role of cancer cell line-based screens to define the impact of cancer genomes on drug response. Curr Opin Genet Dev. 2014;24:114-9.

18. van de Wetering $M$, Francies HE, Francis JM, Bounova G, lorio F, Pronk A, van Houdt W, van Gorp J, Taylor-Weiner A, Kester L, et al. Prospective derivation of a living organoid biobank of colorectal cancer patients. Cell. 2015;161:933-45.

19. Lee JK, Liu Z, Sa JK, Shin S, Wang J, Bordyuh M, Cho HJ, Elliott O, Chu T, Choi SW, et al. Pharmacogenomic landscape of patient-derived tumor cells informs precision oncology therapy. Nat Genet. 2018;50:1399-411.

20. Cancer Genome Atlas Research N. Integrated genomic analyses of ovarian carcinoma. Nature. 2011;474:609-15.

21. Cancer Genome Atlas Research N, Albert Einstein College of M, Analytical Biological S, Barretos Cancer H, Baylor College of M, Beckman Research Institute of City of H, Buck Institute for Research on A, Canada's Michael Smith Genome Sciences C, Harvard Medical S, Helen FGCC, et al. Integrated genomic and molecular characterization of cervical cancer. Nature. 2017;543:378-84.

22. Cancer Genome Atlas Research N, Kandoth C, Schultz N, Cherniack AD, Akbani R, Liu Y, Shen H, Robertson AG, Pashtan I, Shen R, et al. Integrated genomic characterization of endometrial carcinoma. Nature. 2013;497:67-73.

23. Berger AC, Korkut A, Kanchi RS, Hegde AM, Lenoir W, Liu W, Liu Y, Fan H, Shen H, Ravikumar $\mathrm{V}$, et al. A comprehensive pan-cancer molecular study of gynecologic and breast cancers. Cancer Cell. 2018;33:690-705 e699.

24. Shin HT, Choi YL, Yun JW, Kim NKD, Kim SY, Jeon HJ, Nam JY, Lee C, Ryu D, Kim SC, et al. Prevalence and detection of low-allele-fraction variants in clinical cancer samples. Nat Commun. 2017:8:1377.

25. Philip CA, Laskov I, Beauchamp MC, Marques M, Amin O, Bitharas J, Kessous R, Kogan L, Baloch T, Gotlieb WH, Yasmeen A. Inhibition of PI3K-AKT-mTOR pathway sensitizes endometrial cancer cell lines to PARP inhibitors. BMC Cancer. 2017;17:638

26. Slomovitz BM, Coleman RL. The PI3K/AKT/mTOR pathway as a therapeutic target in endometrial cancer. Clin Cancer Res. 2012;18:5856-64.

27. Dellinger TH, Planutis K, Tewari KS, Holcombe RF. Role of canonical Wnt signaling in endometrial carcinogenesis. Expert Rev Anticancer Ther. 2012; 12:51-62.

28. Coopes A, Henry CE, Llamosas E, Ford CE. An update of Wnt signalling in endometrial cancer and its potential as a therapeutic target. Endocr Relat Cancer. 2018;25:647-62.

29. House CD, Hernandez L, Annunziata CM. In vitro enrichment of ovarian cancer tumor-initiating cells. J Vis Exp. 2015;96:e52446.
30. Sun Y, Yoshida T, Okabe M, Zhou K, Wang F, Soko C, Saito S, Nikaido T. Isolation of stem-like cancer cells in primary endometrial cancer using cell surface markers CD133 and CXCR4. Transl Oncol. 2017;10:976-87.

31. Fallahi-Sichani M, Honarnejad S, Heiser LM, Gray JW, Sorger PK. Metrics other than potency reveal systematic variation in responses to cancer drugs. Nat Chem Biol. 2013;9:708-14.

32. Jang IS, Neto EC, Guinney J, Friend SH, Margolin AA. Systematic assessment of analytical methods for drug sensitivity prediction from cancer cell line data. Pac Symp Biocomput. 2014:63-74.

33. Huang S, Pang L. Comparing statistical methods for quantifying drug sensitivity based on in vitro dose-response assays. Assay Drug Dev Technol. 2012;10:88-96.

34. Heo EJ, Cho YJ, Cho WC, Hong JE, Jeon HK, Oh DY, Choi YL, Song SY, Choi JJ, Bae DS, et al. Patient-derived xenograft models of epithelial ovarian cancer for preclinical studies. Cancer Res Treat. 2017:49:915-26.

35. Gao W, Wang M, Wang L, Lu H, Wu S, Dai B, Ou Z, Zhang L, Heymach JV, Gold KA, et al. Selective antitumor activity of ibrutinib in EGFR-mutant nonsmall cell lung cancer cells. J Natl Cancer Inst. 2014;106(9).

36. Slomovitz BM, Jiang Y, Yates MS, Soliman PT, Johnston T, Nowakowski M, Levenback C, Zhang Q, Ring K, Munsell MF, et al. Phase II study of everolimus and letrozole in patients with recurrent endometrial carcinoma. J Clin Oncol. 2015;33:930-6.

37. Meinhold-Heerlein I, Fotopoulou C, Harter P, Kurzeder C, Mustea A, Wimberger P, Hauptmann S, Sehouli J. The new WHO classification of ovarian, fallopian tube, and primary peritoneal cancer and its clinical implications. Arch Gynecol Obstet. 2016;293:695-700.

38. Cole AJ, Dwight T, Gill AJ, Dickson KA, Zhu Y, Clarkson A, Gard GB, Maidens J, Valmadre S, Clifton-Bligh R, Marsh DJ. Assessing mutant p53 in primary high-grade serous ovarian cancer using immunohistochemistry and massively parallel sequencing. Sci Rep. 2016;6:26191.

39. Rechsteiner M, Zimmermann AK, Wild PJ, Caduff R, von Teichman A, Fink D, Moch $\mathrm{H}$, Noske A. TP53 mutations are common in all subtypes of epithelial ovarian cancer and occur concomitantly with KRAS mutations in the mucinous type. Exp Mol Pathol. 2013;95:235-41.

40. Kuo KT, Mao TL, Jones S, Veras E, Ayhan A, Wang TL, Glas R, Slamon D, Velculescu VE, Kuman RJ, Shih le M. Frequent activating mutations of PIK3CA in ovarian clear cell carcinoma. Am J Pathol. 2009;174:1597-601.

41. Chandler RL, Damrauer JS, Raab JR, Schisler JC, Wilkerson MD, Didion JP, Starmer J, Serber D, Yee D, Xiong J, et al. Coexistent ARID1A-PIK3CA mutations promote ovarian clear-cell tumorigenesis through protumorigenic inflammatory cytokine signalling. Nat Commun. 2015;6:6118.

42. TCGA. Integrated genomic analyses of ovarian carcinoma. Nature. 2011;474: 609-15

43. Robson M, Goessl C, Domchek S. Olaparib for metastatic germline BRCAmutated breast cancer. N Engl J Med. 2017;377:1792-3.

44. Moore K, Colombo N, Scambia G, Kim BG, Oaknin A, Friedlander M, Lisyanskaya A, Floquet A, Leary A, Sonke GS, et al. Maintenance olaparib in patients with newly diagnosed advanced ovarian cancer. N Engl J Med. 2018;379:2495-505.

45. Dong P, Tada M, Hamada J, Nakamura A, Moriuchi T, Sakuragi N. p53 dominant-negative mutant $\mathrm{R} 273 \mathrm{H}$ promotes invasion and migration of human endometrial cancer HHUA cells. Clin Exp Metastasis. 2007;24:471-83.

46. Cancer Genome Atlas Research N. Comprehensive genomic characterization defines human glioblastoma genes and core pathways. Nature. 2008;455: 1061-8.

47. Blank SV, Christos P, Curtin JP, Goldman N, Runowicz CD, Sparano JA, Liebes $\mathrm{L}$, Chen HX, Muggia FM. Erlotinib added to carboplatin and paclitaxel as first-line treatment of ovarian cancer: a phase II study based on surgical reassessment. Gynecol Oncol. 2010;119:451-6.

48. Slomovitz BM, Lu KH, Johnston T, Coleman RL, Munsell M, Broaddus RR, Walker C, Ramondetta LM, Burke TW, Gershenson DM, Wolf J. A phase 2 study of the oral mammalian target of rapamycin inhibitor, everolimus, in patients with recurrent endometrial carcinoma. Cancer. 2010;116:5415-9.

49. Colombo N, McMeekin DS, Schwartz PE, Sessa C, Gehrig PA, Holloway R, Braly P, Matei D, Morosky A, Dodion PF, et al. Ridaforolimus as a single agent in advanced endometrial cancer: results of a single-arm, phase 2 trial. $\mathrm{Br}$ J Cancer. 2013;108:1021-6.

50. Tsoref D, Welch S, Lau S, Biagi J, Tonkin K, Martin LA, Ellard S, Ghatage P, Elit L, Mackay HJ, et al. Phase II study of oral ridaforolimus in women with recurrent or metastatic endometrial cancer. Gynecol Oncol. 2014;135:184-9. 
51. Liu JF, Barry WT, Birrer M, Lee JM, Buckanovich RJ, Fleming GF, Rimel B, Buss MK, Nattam S, Hurteau J, et al. Combination cediranib and olaparib versus olaparib alone for women with recurrent platinum-sensitive ovarian cancer: a randomised phase 2 study. Lancet Oncol. 2014;15:1207-14.

52. Ledermann JA, Embleton AC, Raja F, Perren TJ, Jayson GC, Rustin GJS, Kaye SB, Hirte H, Eisenhauer E, Vaughan M, et al. Cediranib in patients with relapsed platinum-sensitive ovarian cancer (ICON6): a randomised, doubleblind, placebo-controlled phase 3 trial. Lancet. 2016;387:1066-74.

53. Zilfou JT, Lowe SW. Tumor suppressive functions of p53. Cold Spring Harb Perspect Biol. 2009;1:a001883.

54. Ko HL, Ren EC. Functional aspects of PARP1 in DNA repair and transcription. Biomolecules. 2012;2:524-48.

55. Williamson CT, Kubota E, Hamill JD, Klimowicz A, Ye R, Muzik H, Dean M, Tu L, Gilley D, Magliocco AM, et al. Enhanced cytotoxicity of PARP inhibition in mantle cell lymphoma harbouring mutations in both ATM and p53. EMBO Mol Med. 2012;4:515-27.

56. Bjorge JD, Jakymiw A, Fujita DJ. Selected glimpses into the activation and function of Src kinase. Oncogene. 2000;19:5620-35.

57. Trimble EL. The NIH Consensus Conference on Ovarian Cancer: screening, treatment, and follow-up. Gynecol Oncol. 1994;55:S1-3.

58. Shah AN, Gallick GE. Src, chemoresistance and epithelial to mesenchymal transition: are they related? Anti-Cancer Drugs. 2007:18:371-5.

59. Ruzinova MB, Benezra R. Id proteins in development, cell cycle and cancer. Trends Cell Biol. 2003:13:410-8.

60. Lee SB, Frattini V, Bansal M, Castano AM, Sherman D, Hutchinson K, Bruce JN, Califano A, Liu G, Cardozo T, et al. An ID2-dependent mechanism for VHL inactivation in cancer. Nature. 2016;529:172-7.

61. Fong S, Debs RJ, Desprez PY. Id genes and proteins as promising targets in cancer therapy. Trends Mol Med. 2004;10:387-92.

62. Rollin J, Blechet C, Regina S, Tenenhaus A, Guyetant S, Gidrol X. The intracellular localization of ID2 expression has a predictive value in non small cell lung cancer. PLoS One. 2009;4:e4158.

63. Sa JK, Hwang JR, Cho YJ, Ryu JY, Choi JJ, Jeong SY, Kim J, Kim MS, Paik ES, Lee YY, Choi CH, Kim TJ, Kim BG, Bae DS, Lee Y, Her NG, Shin YJ, Cho HJ, Kim JY, Seo YJ, Koo H, Oh JW, Lee T, Kim HS, Song SY, Bae JS, Park WY, Han HD, Ahn HJ, Sood AK, Rabadan R, Lee JK, Nam DH, Lee JW. Gynecologic cancer genome. European Genome-phenome Archive. 2019. https://www. ebi.ac.uk/ega/studies/EGAS00001003556. Accessed 17 Nov 2019

\section{Publisher's Note}

Springer Nature remains neutral with regard to jurisdictional claims in published maps and institutional affiliations.

Ready to submit your research? Choose BMC and benefit from:

- fast, convenient online submission

- thorough peer review by experienced researchers in your field

- rapid publication on acceptance

- support for research data, including large and complex data types

- gold Open Access which fosters wider collaboration and increased citations

- maximum visibility for your research: over $100 \mathrm{M}$ website views per year

At $\mathrm{BMC}$, research is always in progress.

Learn more biomedcentral.com/submissions 